\title{
Dípteros acuáticos del departamento del Chocó, Colombia
}

\section{Aquatic diptera in the department of Chocó, Colombia}

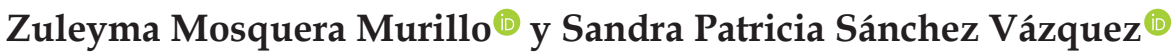

\section{Resumen}

Se presenta un reporte preliminar sobre la riqueza y distribución de los dípteros acuáticos en el departamento del Chocó, a partir de 490 especímenes (larvas y pupas) de la Colección Limnológica del Chocó (CLCH-Insec, Universidad Tecnológica del Chocó), que corresponden a 18 municipios (entre 5 y 3202 m s.n.m.), 46 corrientes hídricas de diferente orden y 14 ecosistemas lénticos de las cuencas de los ríos Atrato, San Juan y Baudó. Se reportan 12 familias y 30 géneros, de los cuales 4 familias y 16 géneros, son registrados por primera vez para el Chocó. Chironomidae es la familia más abundante y más ampliamente distribuida, mientras que Dixidae y Sciomyzidae son las familias de menor abundancia y distribución. La mayor riqueza taxonómica la registran las familias Culicidae y Ceratopogonidae, con cinco géneros cada una. Las macrófitas son el sustrato con mayor riqueza específica. El mayor registro taxonómico se encuentra en la cuenca del río Atrato, con 11 familias y 24 géneros. Estos resultados permiten evidenciar la enorme diversidad de los dípteros acuáticos en el departamento del Chocó y la necesidad de adelantar nuevas investigaciones que amplíen su conocimiento taxonómico y ecológico en la región.

Palabras clave. CLCH. Diptera. Ecosistemas acuáticos. Insecta.

\begin{abstract}
We present a preliminary report on the richness and distribution of aquatic Diptera in the department of Chocó, from 490 specimens (larvae and pupae) kept in the Chocó Limnological Collection (CLCH-Insec), corresponding to 18 municipalities (between 5 and 3202 m a.s.l.), 46 water streams of different order and 14 lentic ecosystems of the Atrato, San Juan and Baudó Rivers basins. There are 12 families and 30 genera, of which 4 families and 16 genera are recorded for the first time for Chocó. Chironomidae is the most abundant and most widely distributed family, while Dixidae and Sciomyzidae are the families with lowest abundance and distribution. The highest taxonomic richness is recorded for the families Culicidae and Ceratopogonidae, with five genera each. Macrophytes are the substrate with the greatest specific richness. The largest taxonomic record is found in the Atrato River basin, with 11 families and 24 genera. These results show the enormous diversity of aquatic Diptera in the department of Chocó and the need to develop new research that will broaden their taxonomic and ecological knowledge in the region..
\end{abstract}

Keywords. CLCH. Diptera. aquatic ecosystems. Insecta. 


\section{Introducción}

Los dípteros constituyen uno de los órdenes de insectos más complejos, abundantes y ampliamente distribuidos en el mundo, representado por aproximadamente 153000 especies distribuidas en más de 180 familias, de las cuales 118, con 31000 especies, están reportadas en la región neotropical (Lizarralde, 2009; Amorim, 2009).

Las larvas de la mayoría de las especies pueden ser consideradas como acuáticas en un sentido amplio, pues para sobrevivir deben estar en ambientes desde ligeramente húmedos a húmedos, como dentro de tejidos de plantas vivas, materia orgánica en descomposición, parásitos de otros animales o en asociación con cuerpos de agua; solo las especies que ocupan este último hábitat se consideran acuáticas (Courtney \& Merritt, 2008; DeWalt et al., 2010).

Las larvas de dípteros tienen la capacidad de vivir en hábitats muy variados, desde ríos, arroyos, lagos, embalses, brácteas de bromeliáceas y demás plantas que acumulan agua, en orificios de troncos viejos y en las costas marinas (Roldán \& Ramírez, 2008). Presentan gran capacidad de colonización de los sustratos, gracias a sus diferentes variaciones morfológicas, y además de ser parte de la dieta de la biota acuática, ayudan al reciclaje de nutrientes sedimentados y a la transformación de la materia orgánica de los fondos, mediante los movimientos ondulatorios de sus cuerpos (Courtney et al., 2009).

Por otra parte, algunos dípteros (Culicidae, Glossinidae, Psychodidae, Simuliidae) son de gran interés en salud pública ya que son vectores biológicos de patógenos o pueden producir reacciones alérgicas (Chironomidae) (McCafferty; 1983; Merritt et al., 2009). También aportan información importante en estudios y análisis de la estructura y función de ecosistemas acuáticos, adaptaciones ecológicas y estructurales, análisis de calidad de agua, biomonitoreo y en biología de la conservación (Courtney \& Merritt, 2008).
En Colombia son importantes contribuciones al conocimiento los trabajos sobre dípteros acuáticos relacionados con ecología, riqueza, sistemática y medicina forense de Wolff et al. (1991), Pinilla (2000), Ruiz et al. (2000), Miranda-Esquivel \& Coscarón (2001), Pérez (2007), Rojas-Sandino et al. (2018), Oviedo-Machado \& Reinoso-Flores (2018), Rojas-Céspedes et al. (2018). En el departamento del Chocó, los estudios sobre el orden son aún incipientes, reportándose de forma fragmentaria la presencia de 8 familias y 14 géneros (Mosquera \& Córdoba, 2015a; Mosquera \& Córdoba, 2015b; Mosquera \& Mosquera, 2017; Mosquera, 2017; Mosquera, 2018).

El presente artículo tiene como objetivo aportar información sobre la riqueza genérica y distribución de los dípteros acuáticos en el departamento del Chocó, a partir de la revisión de los especímenes que se encuentran depositados en la Colección Limnológica del Chocó (CLCH-Insec), de la Universidad Tecnológica del Chocó, en Quibdó.

\section{Materiales y métodos}

La información taxonómica que se presenta es el resultado de la recopilación y revisión de información sobre larvas y pupas del orden Diptera depositadas en la Colección Limnológica del Chocó «CLCH-Insec» (Universidad Tecnológica del Chocó) https:/ /ipt.biodiversidad.co/sib/ resource?r=utch-002, conservadas y preservadas en su totalidad en alcohol al $80 \%$. Estos organismos fueron recolectados a través de proyectos de grado e investigaciones realizadas por el grupo de Limnología de la Universidad Tecnológica del Chocó, entre los años 2004 y 2018, y su información fue validada para verificar los datos de coordenadas, distribución altitudinal y procedencia. Los especímenes del orden Diptera estudiados corresponden a estadíos inmaduros recolectados en diferentes sustratos sumergidos, como rocas del lecho, hojarasca, troncos, vegetación ribereña, musgo y macrófitas. La determinación taxonómica se realizó en 
el laboratorio de Limnología de la UTCH, para lo que se emplearon las claves de Borkent \& Spinelli (2007), Merrit et al. (2008), Domínguez \& Fernández (2009) y Trivinho (2011), con la ayuda de un estereomicroscopio NIKON SMZ 745. Para la identificación de las larvas de quironómidos se realizaron preparados permanentes en Euparal (Trivinho, 2011).

\section{Resultados}

Riqueza faunística y abundancia relativa de dípteros acuáticos. Los datos de los 490 ejemplares revisados (98.16 \% larvas y $1.83 \%$ pupas) permiten reportar de forma preliminar para el departamento del Chocó 2 subórdenes, 12 familias y 30 géneros de dípteros acuáticos (Tabla 1),

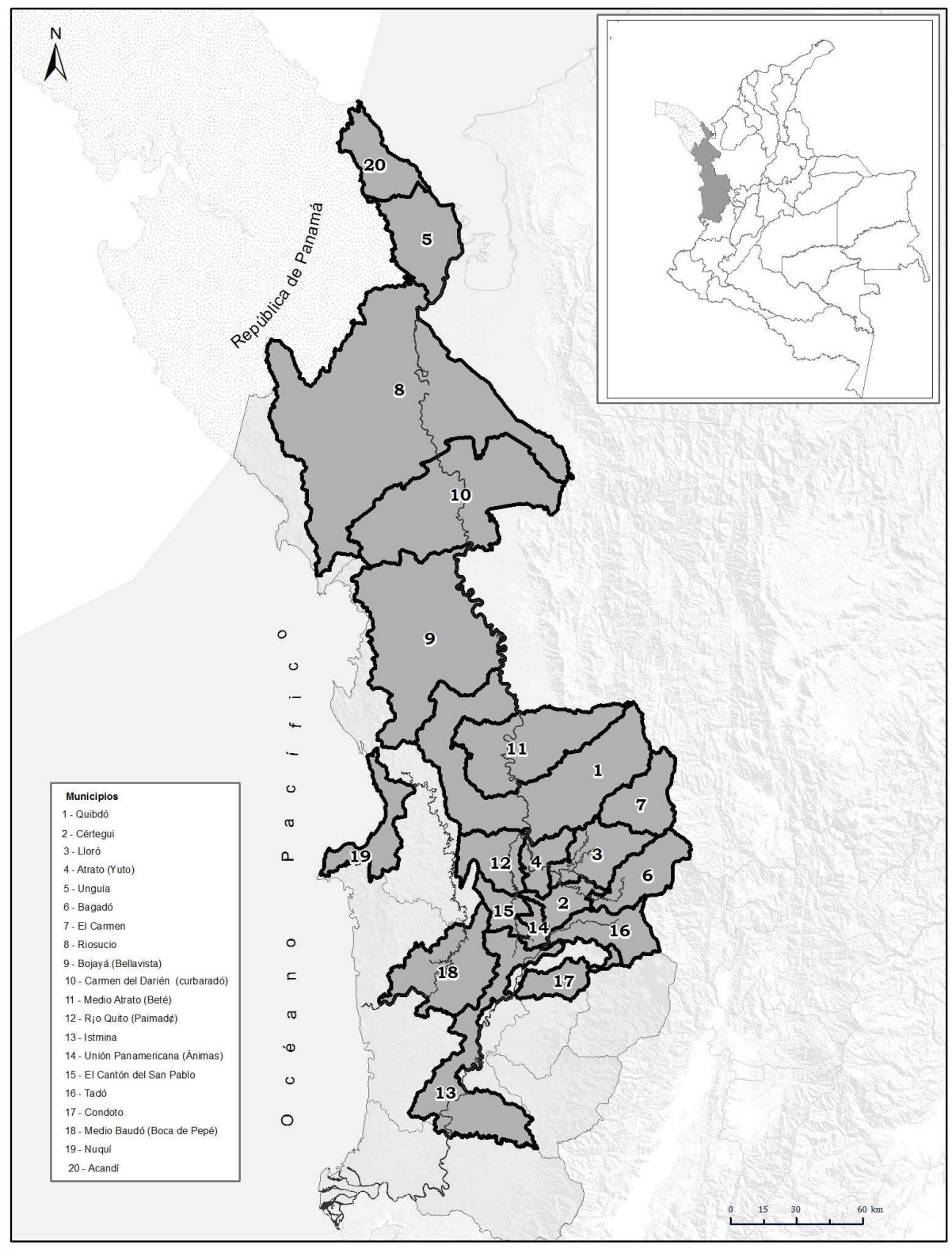

Figura 1. División política del departamento de Chocó, Colombia con las áreas de distribución del orden Diptera. Base cartográfica: IGAC Instituto Geográfico Agustín Codazzi. 
Tabla 1. Listado taxonómico, abundancia relativa, etapa de vida y reporte de nuevos géneros del orden Diptera para el departamento del Chocó, depositados en la Colección Limnológica del Chocó (CLCH-Insec), Quibdó, Colombia.

\begin{tabular}{|c|c|c|c|}
\hline Taxón & Abundancia relativa $\%$ & Estadio & Registro \\
\hline \multicolumn{4}{|c|}{ Suborden Nematocera } \\
\hline \multicolumn{4}{|l|}{ Chironomidae } \\
\hline Chironomus & 15.92 & Larva, Pupa & \\
\hline Ablabesmyia & 38.57 & Larva & \\
\hline Orthocladius & 0.81 & Larva, Pupa & \\
\hline Pentaneura & 4.08 & Larva & \\
\hline \multicolumn{4}{|l|}{ Simuliidae } \\
\hline Simulium & 16.73 & Larva & \\
\hline \multicolumn{4}{|c|}{ Ceratopogonidae } \\
\hline Bezzia & 0.61 & Larva & \\
\hline Stilobezzia & 0.61 & Larva & Nuevo \\
\hline Probezzia & 0.20 & Larva & Nuevo \\
\hline Dasyhelea & 0.82 & Larva & Nuevo \\
\hline Culicoides & 0.61 & Pupa & Nuevo \\
\hline \multicolumn{4}{|l|}{ Limoniidae } \\
\hline Hexatoma & 5.31 & Larva & \\
\hline Dactylolabis & 2.65 & Larva & Nuevo \\
\hline Geranomyia & 0.82 & Larva & Nuevo \\
\hline Limonia & 0.41 & Larva & Nuevo \\
\hline \multicolumn{4}{|l|}{ Psychodidae } \\
\hline Psychoda & 0.82 & Larva, Pupa & Nuevo \\
\hline Pericoma & 0.61 & Larva & Nuevo \\
\hline Clogmia & 0.20 & Larva & Nuevo \\
\hline Maruina & 0.20 & Larva & \\
\hline \multicolumn{4}{|l|}{ Culicidae } \\
\hline Culiseta & 0.20 & Larva & Nuevo \\
\hline Chagasia & 2.04 & Larva & \\
\hline Culex & 0.41 & Larva & \\
\hline Mansonia & 1.22 & Larva & \\
\hline Orthopodomyia & 0.41 & Larva & \\
\hline \multicolumn{4}{|l|}{ Chaoboridae } \\
\hline Chaoborus & 4.08 & Larva & \\
\hline Eucorethra & 0.20 & Larva & Nuevo \\
\hline Dixidae & & & Nuevo \\
\hline Dixella & 0.20 & Larva & Nuevo \\
\hline Athericidae & & & Nuevo \\
\hline Atherix & 0.20 & Larva & Nuevo \\
\hline
\end{tabular}


Suborden Brachycera

\begin{tabular}{llll}
\hline Dolichopodidae & & Nuevo \\
\hline Aphrosylus & 0.41 & Larva & Nuevo \\
\hline Sciomyzidae & & Larva & Nuevo \\
\hline Pherbellia & 0.20 & & Nuevo \\
\hline Tabanidae & & Larva & \\
\hline Tabanus & 0.41 & & \\
\hline
\end{tabular}

los cuales se encontraron asociados a 46 corrientes hídricas de diferente orden y 13 ecosistemas lénticos de las cuencas de los ríos Atrato, San Juan y Baudó, ubicados en 18 municipios del departamento (Figura 1). Estos resultados incrementan el número conocido de taxones distribuidos en el departamento, puesto que 4 familias (33.33 \%) y 16 géneros $(53.33 \%$ ) son reportados por primera vez para el Chocó (Tabla 1).

De las 12 familias del orden Diptera registradas en la Colección Limnológica del Chocó, Chironomidae es la que presenta el mayor número de organismos, con el $59.39 \%$, seguida de Simuliidae con un $16.73 \%$. Las restantes familias presentan abundancias inferiores al $10 \%$; sin embargo, Culicidae y Ceratopogonidae son las que presentan la mayor riqueza con cinco géneros cada una (Figura 2).

Distribución de los dípteros acuáticos. A nivel de las principales cuencas del departamento del Chocó, es la del río Atrato la que registra el mayor número de individuos de la colección con el 70.81 $\%$, representados en 11 familias y 24 géneros; en segundo lugar se encuentra la cuenca del río San Juan con el $23.46 \%$, con 5 familias y 9 géneros, y en último lugar, la cuenca del río Baudó con el 3.87 \%, con 2 familias y 3 géneros. Cuatro familias y cinco géneros se registran en microcuencas pericontinentales que fluyen directamente a los océanos Atlántico y Pacífico (Tabla 2).

Chironomidae es la familia con mayor distribución en la colección, al encontrarse en el $77.7 \%$ de los municipios (14), seguida de Simuliidae y Limoniidae con el 50 \% (9); mientras que Dolichopodidae,
Sciomycidae, Athericidae y Dixidae se registran en solo uno de los municipios representados en la misma (Tabla 2, Figura 3).

Desde el punto de vista altitudinal, los géneros que presentaron los rangos más amplios fueron Geranomyia, Ablabesmyia y Simulium; sin embargo, para todos los géneros reportados se amplía el rango de distribución altitudinal en el país, al registrarse en localidades por debajo de los $100 \mathrm{~m} \mathrm{~s}$. n. m. (Tabla 2). Es importante destacar que 17 géneros (54.83\%) solo se registran en una de las localidades de estudio; por lo tanto, su valor en la distribución altitudinal fue puntual.

Los ejemplares revisados del orden Diptera se encontraron asociados a siete sustratos: piedra, hojarasca, tronco, vegetación ribereña, macrófitas, musgo, sedimento, siendo las macrófitas el que registra la mayor riqueza de géneros (13), seguido de piedra (12), hojarasca (9), vegetación ribereña (7), sedimento (5), musgo (5) y finalmente tronco (2) (Tabla 2).

\section{Discusión}

\section{Riqueza faunística y abundancia relativa de dípteros acuáticos.}

Los ejemplares del orden Diptera presentes en la Colección Limnológica del Chocó constituyen un aporte importante al conocimiento de la diversidad regional de este grupo, ya que se reportan familias y géneros por primera vez para la región (Tabla 1). 
- Abundancia relativa $\Delta$ No. de géneros

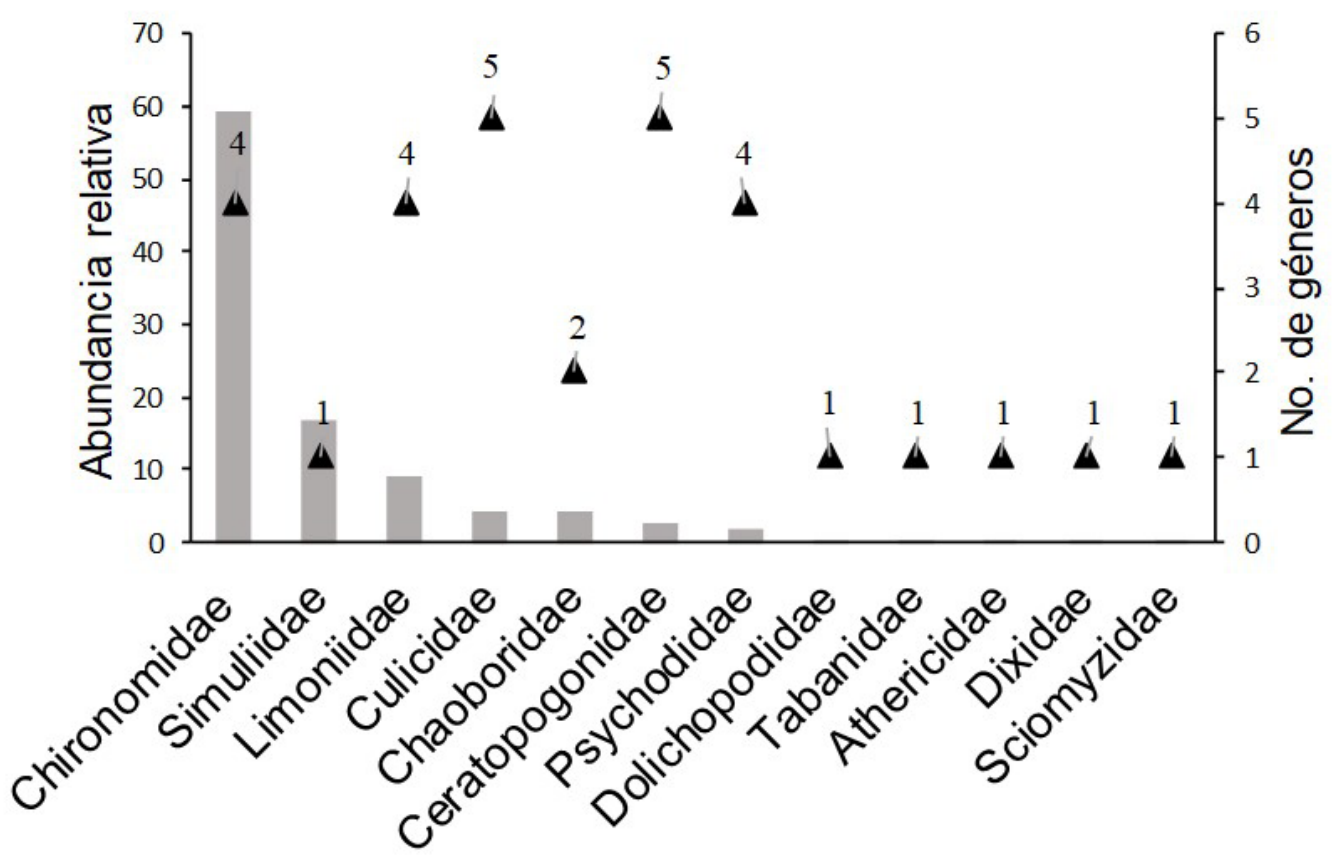

Figura 2. Abundancia relativa y riqueza genérica de familias de dípteros acuáticos registradas en la Colección Limnológica del Chocó (CLCH-Insec), Quibdó, Colombia.

Tabla 2. Distribución de las familias y géneros de dípteros acuáticos en los municipios del Chocó, Colombia, ubicación por macrocuenca y rango de altitud.

Distribución: Ac, Acandí; At, Atrato; Ba, Bagadó; Bo, Bojayá; Bs, Bahía solano; Ca, Carmen de Atrato; Cd, Carmen del Darién; Cdp, Cantón de San Pablo; Ce, Cértegui; Co, Condoto; Is, Istmina; Ma, Medio Atrato; Mb, Medio Baudó; Qb, Quibdó; Rs, Río Sucio; Ta, Tadó; Un, Unguía; Up, Unión Panamericana. Sustrato: H, hojarasca; Ma, macrófitas; Mu, musgo; P, piedra; Se, sedimento T, tronco; Vr. vegetación ribereña. +, microcuenca pericontinental.

\begin{tabular}{|c|c|c|c|c|}
\hline Taxón & $\begin{array}{c}\text { Distribución en el } \\
\text { Chocó }\end{array}$ & Cuenca & Sustrato & $\begin{array}{l}\text { Rango de altituc } \\
\text { (m. s. n. m.) }\end{array}$ \\
\hline \multicolumn{5}{|l|}{ Chironomidae } \\
\hline Chironomus & Qb, Un, Up, Is & $\begin{array}{c}\text { Atrato, San Juan, } \\
\text { Pacífica }^{+}\end{array}$ & $\mathrm{P}, \mathrm{H}, \mathrm{Se}$ & $46-190$ \\
\hline Ablabesmyia & $\begin{array}{l}\mathrm{Qb}, \mathrm{Un}, \mathrm{At}, \mathrm{Bo}, \mathrm{Cd}, \mathrm{Rs}, \\
\mathrm{Ca}, \mathrm{Ac}, \mathrm{Up}, \mathrm{Ta}, \mathrm{Cdp}, \mathrm{Is}, \\
\mathrm{Mb}, \mathrm{Ce}\end{array}$ & $\begin{array}{l}\text { Atrato, San Juan, } \\
\text { Baudó, Pacífica + }\end{array}$ & $\mathrm{P}, \mathrm{H}, \mathrm{Vr}, \mathrm{Ma}, \mathrm{T}, \mathrm{Se}, \mathrm{Mu}$ & $5-2247$ \\
\hline Orthocladius & $\mathrm{Qb}$ & Atrato & $\mathrm{Ma}, \mathrm{Mu}$ & $32-90$ \\
\hline Pentaneura & $\begin{array}{l}\mathrm{Qb}, \mathrm{Un}, \mathrm{Bo}, \mathrm{At}, \mathrm{Cd}, \mathrm{Rs}, \\
\mathrm{Ta}, \mathrm{Is}, \mathrm{Mb}\end{array}$ & $\begin{array}{c}\text { Atrato, San Juan, } \\
\text { Baudó }\end{array}$ & $\mathrm{H}, \mathrm{Vr}, \mathrm{Ma}$ & $5-133$ \\
\hline
\end{tabular}




\begin{tabular}{|c|c|c|c|c|}
\hline \multicolumn{5}{|l|}{ Simuliidae } \\
\hline Simulium & $\begin{array}{l}\mathrm{Qb}, \mathrm{At}, \mathrm{Ca}, \mathrm{Ta}, \mathrm{Cdp}, \mathrm{Mb}, \mathrm{Is} \text {, } \\
\mathrm{Co}, \mathrm{Up}\end{array}$ & $\begin{array}{l}\text { Atrato, San Juan, } \\
\text { Baudó }\end{array}$ & $\mathrm{P}, \mathrm{H}, \mathrm{Vr}, \mathrm{Se}$ & $32-2180$ \\
\hline \multicolumn{5}{|l|}{ Ceratopogonidae } \\
\hline Bezzia & $\mathrm{Qb}$ & Atrato & $\mathrm{Se}, \mathrm{Vr}$ & $50-90$ \\
\hline Stilobezzia & Qb, Un, Rs & Atrato, Pacífica ${ }^{+}$ & $\mathrm{P}, \mathrm{Ma}$ & $8-190$ \\
\hline Probezzia & Bo & Atrato & $\mathrm{Ma}$ & 36 \\
\hline Culicoides & $\mathrm{Qb}$ & Atrato & $\mathrm{Ma}$ & 43 \\
\hline Dasyhelea & $\mathrm{Qb}$ & Atrato & $\mathrm{P}$ & 57 \\
\hline \multicolumn{5}{|l|}{ Dolichopodidae } \\
\hline Aphrosylus & $\mathrm{Qb}$ & Atrato & $\mathrm{P}$ & 90 \\
\hline \multicolumn{5}{|l|}{ Limoniidae } \\
\hline Hexatoma & $\mathrm{Qb}, \mathrm{At}, \mathrm{Ce}, \mathrm{Up}, \mathrm{Ta}, \mathrm{Is}, \mathrm{Ac}$ & $\begin{array}{c}\text { Atrato, San Juan, } \\
\text { Pacífica }^{+}\end{array}$ & $\mathrm{P}, \mathrm{H}, \mathrm{Vr}$ & $56-133$ \\
\hline Dactylolabis & $\mathrm{Qb}, \mathrm{Up}$ & Atrato, San Juan & $\mathrm{H}, \mathrm{T}$ & $47-103$ \\
\hline Geranomyia & $\mathrm{Ba}, \mathrm{Bs}$ & Atrato, Pacífica ${ }^{+}$ & $\mathrm{P}$ & $152-3202$ \\
\hline Limonia & $\mathrm{Ta}$ & San Juan & $\mathrm{H}$ & 135 \\
\hline \multicolumn{5}{|l|}{ Psychodidae } \\
\hline Psychoda & $\mathrm{Qb}$ & Atrato & $\mathrm{P}, \mathrm{Mu}$ & $46-90$ \\
\hline Pericoma & $\mathrm{Qb}$ & Atrato & $\mathrm{Mu}$ & 90 \\
\hline Clogmia & $\mathrm{Qb}$ & Atrato & $\mathrm{Mu}$ & 47 \\
\hline Maruina & Up & San Juan & $\mathrm{Vr}$ & 103 \\
\hline \multicolumn{5}{|l|}{ Culicidae } \\
\hline Culiseta & $\mathrm{Qb}$ & Atrato & Ma & 50 \\
\hline Chagasia & $\mathrm{Qb}, \mathrm{Un}$ & Atrato & $\mathrm{Ma}$ & $5-32$ \\
\hline Culex & Un & Atrato & Ma & $5-9,3$ \\
\hline Mansonia & Un, Rs & Atrato & $\mathrm{Ma}$ & $5-8$ \\
\hline Orthopodomyia & Un & Atrato & $\mathrm{Ma}$ & 9,3 \\
\hline \multicolumn{5}{|l|}{ Chaoboridae } \\
\hline Chaoborus & $\mathrm{Qb}, \mathrm{Un}, \mathrm{Bo}, \mathrm{Ma}$ & Atrato & $\mathrm{Ma}$, Se & $5-37$ \\
\hline Eucoretha & $\mathrm{Ta}$ & San Juan & $\mathrm{Vr}$ & 95 \\
\hline \multicolumn{5}{|l|}{ Athericidae } \\
\hline Atherix & $\mathrm{Qb}, \mathrm{Un}$ & Atrato & $\mathrm{H}$ & 47 \\
\hline \multicolumn{5}{|l|}{ Tabanidae } \\
\hline Tabanus & $\mathrm{Qb}, \mathrm{Bo}$ & Atrato & $\mathrm{P}, \mathrm{Ma}$ & $26-46$ \\
\hline \multicolumn{5}{|l|}{ Dixidae } \\
\hline Dixella & Un & Pacífica $^{+}$ & $\mathrm{P}$ & 190 \\
\hline \multicolumn{5}{|l|}{ Sciomyzidae } \\
\hline Pherbellia & $\mathrm{Qb}$ & Atrato & $\mathrm{P}$ & 46 \\
\hline
\end{tabular}




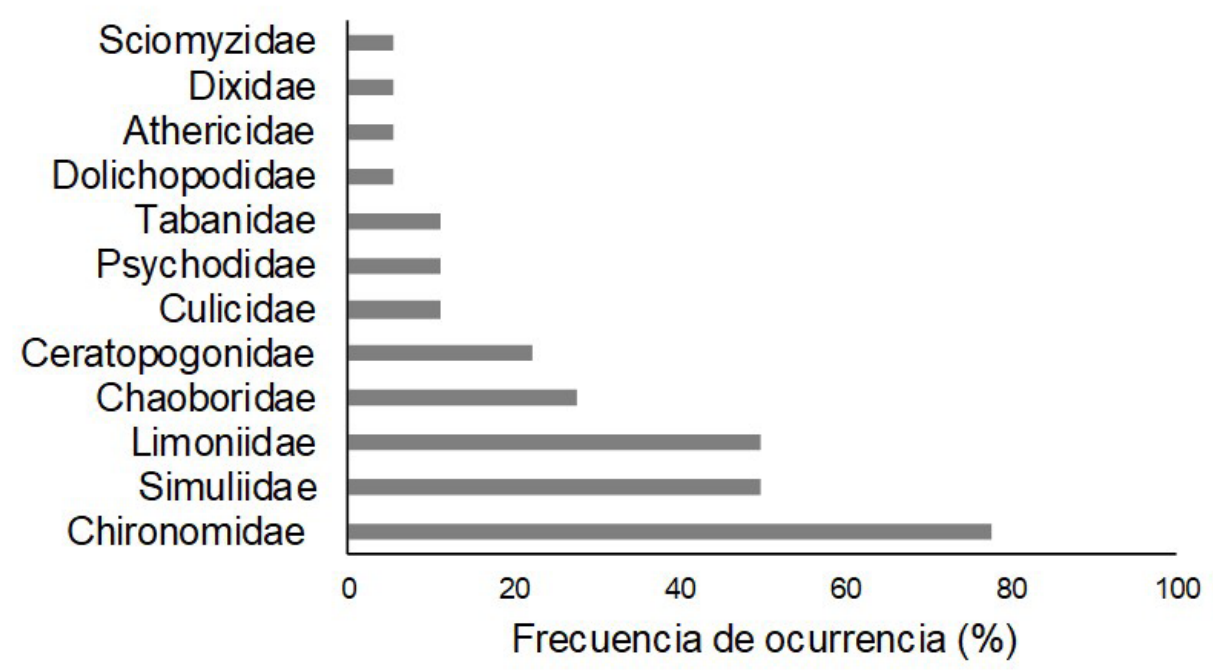

Figura 3. Frecuencia de ocurrencia de familias de dípteros acuáticos en 18 municipios del departamento del Chocó, Colombia.

La abundancia que presentan los chironómidos y simúlidos coincide con otros trabajos realizados en Colombia, como los de Rojas-Céspedes et al. (2018) y Rojas-Sandino et al. (2018) en el departamento del Tolima.

Los dípteros de la familia Chironomidae son los insectos más ampliamente distribuidos y, frecuentemente, los más abundantes en aguas continentales, tanto lóticas como lénticas (Cranston, 1995; Thorp \& Rogers, 2011), donde son de gran importancia en la colonización de cuerpos de agua y juegan un papel fundamental como eslabones en la red trófica (HenriquesOliveira et al., 2003; Rodríguez et al., 2004). Además, gracias a su amplia capacidad de hábitos alimenticios, pueden obtener recursos de cualquier medio y por ende ocupar una gran variedad de microhábitats (DeWalt et al., 2010; Rosa et al., 2013a; Rosa et al., 2013b). Los simúlidos, por su parte, se desarrollan exclusivamente en ambientes acuáticos lóticos desde corrientes temporales hasta los grandes ríos, y, a menudo, dominan la comunidad de macroinvertebrados de estos sistemas lóticos (Adler \& McCreadie, 1997; Kazanci \& Ertunç, 2010). El único género de la familia Simuliidae que se reporta en la Colección es Similium, de gran relevancia, puesto que varias de sus especies tienen importancia desde el punto de vista de la salud humana y animal, ya que son vectores de diferentes agentes patógenos, por lo que son el segundo grupo en importancia médica, después de los Culicidae (Grillet et al., 2005).

Las familias Culicidae y Ceratopogonidae se destacan por su riqueza en los ecosistemas referenciados en la colección. Culicidae es probablemente una de las familias más importantes del orden Diptera debido a que son reservorio y vectores del agente causal de enfermedades tales como el dengue, la fiebre amarilla y la malaria, entre otras, las cuales se transmiten a los humanos y otros animales (González et al., 2016). Particularmente sus larvas y pupas están presentes en una variedad de hábitats lénticos, que incluyen lagos, pantanos, agua salada, agujeros en troncos de árboles, charcas y ríos (Harbach, 2007; Chaverri, 2009). En este estudio se les encontró asociados exclusivamente a las macrófitas presentes en las ciénagas.

Por otro lado, los ceratopogónidos son un grupo de dípteros extremadamente diverso y abundante; sus formas inmaduras se desarrollan en una amplia gama de ambientes húmedos, semiacuático a hábitats totalmente acuáticos, incluyendo ríos y lagos, donde se las puede encontrar en los márgenes y entre los detritus y plantas acuáticas (Wagner et al., 2008). Las de hábitos semiacuáticos, habitan la arena húmeda de playas 
marinas, lodo, pantanos, vegetación húmeda de rocas en lagos, lagunas, corrientes de agua y márgenes de los ríos, aguas salinas y agujeros en troncos de árboles (Sandoval \& Molina, 2000; Borkent et al., 2009).

\section{Distribución de los dípteros acuáticos.}

La mayor abundancia y riqueza de dípteros observada en la zona del río Atrato puede ser atribuida a que es la zona más estudiada del departamento, dada su cercanía a centros poblados y la facilidad de acceso en comparación con las zonas de los ríos San Juan y Baudó, siendo esta última la que presenta mayores dificultades de acceso, lo que ayuda a explicar su baja representación en la colección.

La distribución registrada para la familia Chironomidae concuerda con lo que se reporta para la región neotropical, donde esta familia sobresale por su amplia distribución (Ferrington, 2008; Paggi, 2009; Rosa et al., 2014). Esta puede ser producto de factores medioambientales, como la naturaleza del sustrato, ya que este grupo es capaz de habitar un amplio rango de microhábitats, que incluyen sustrato limo arcilloso, fango, arena, roca, madera sumergida, sedimentos, grava, canto rodado e incluso plantas acuáticas. A su vez, algunos tienen la capacidad de tolerar $\mathrm{pH}$ ácidos, altos grados de salinidad y bajos niveles de oxígeno (Pinder, 1986; Paggi, 2009).

Las familias Dixidae, Athericidae y Sciomyzidae presentan la distribución más restringida, al encontrarse en una única localidad de las registradas en la colección. Las etapas inmaduras de díxidos se desarrollan en los márgenes de los cuerpos de agua, como pantanos, arroyos, ríos, lagos y bromelias (Borkent, 2009), mientras que los atherícidos en su etapa larvaria se encuentran en sistemas lóticos de aguas bien oxigenadas, viviendo en rápidos con fondo rocoso o con grava de ríos o sobre vegetación acuática (McCafferty, 1998; Sandoval \& Molina, 2000; Woodley, 2009). La familia Sciomyzidae, por su parte, comprende especies acuáticas y semiacuáticas y sus larvas están presentes en suelos húmedos de agua dulce y salada, a lo largo de márgenes de lagos y corrientes, especialmente entre la vegetación (McCafferty, 1998).

Según Merrit et al. (2009), los dípteros ocupan un amplio gradiente altitudinal en el Neotrópico, como se evidencia en los ejemplares que reposan en la colección. La altitud afecta la distribución de los macroinvertebrados acuáticos, debido a los cambios de temperatura del agua y contenido de oxígeno (Marchant et al., 1995); particularmente, estas dos variables son señaladas entre los aspectos que más influencia ejercen sobre la distribución, abundancia y riqueza de los insectos acuáticos, debido a que están relacionados con los procesos metabólicos de los organismos aeróbicos y la productividad biológica (Ocon et al., 2004; Domínguez \& Fernández, 2009).

Los dípteros que reposan en la Colección Limnológica se encontraron asociados a una gran variedad de sustratos, sobresaliendo las macrófitas y las piedras. Según Sandoval \& Molina (2000) los dípteros son posiblemente el grupo más ampliamente adaptado de todos los insectos acuáticos, lo que se evidencia en la gran variedad de hábitats que ocupan sus estados preimaginales, superior a la de cualquier otro orden de insectos; de allí que sus larvas y pupas puedan ser encontradas en un gran número de sustratos, tanto en sistemas lóticos como lénticos (Lizarralde, 2009).

Los resultados de este trabajo contribuyen al conocimiento de la riqueza genérica y distribución de los dípteros acuáticos en el departamento del Chocó y en el país, poniendo además en evidencia la enorme diversidad del orden en la región, por lo que se espera que, con un aumento de los estudios taxonómicos de la fauna de dípteros, el número de taxones registrados se incremente considerablemente.

\section{Agradecimientos}

Agradecemos al laboratorio de Limnología, a la Vicerrectoría de Investigaciones y al Centro de Investigación en Biodiversidad y Hábitat "CEIBHA" de 
la Universidad Tecnológica del Chocó, por su apoyo para la organización y creación de la Colección Limnológica del Chocó, a partir de la cual se generó la información que se presenta en este artículo. A las biólogas Karen E. Córdoba y Yasiris Salas Tovar (Q.E.P.D.) y a la estudiante Karen Palomeque, por su participación en la organización de la colección y trabajo de laboratorio. Agradecemos igualmente, a los revisores anónimos por sus comentarios y sugerencias, los cuales contribuyeron a mejorar la versión final del manuscrito.

\section{Referencias}

Adler, P. H. \& McCreadie, J. W. (1997). Insect life: the hidden ecology of black flies: sibling species and ecological scale. American Entomologist, 43(3), 153-162. https://doi.org/10.1093/ae/43.3.153

Amorim, D. S. (2009). Neotropical Diptera diversity: richness, patterns, and perspectives. En Pape, T., Bickel, D. \& Meier, R. (Eds.). Diptera diversity: status, challenges and tools. Pp. 71-97. Leiden: Koninklijke Brill NV. https://doi.org/10.1163/ej.9789004148970.I-459.17

Borkent, A. \& Spinelli, R. C. (2007). Neotropical Ceratopogonidae (Diptera: Insecta). En Adis, J., Arias, J. R., Rueda-Delgado, G. \& Wattzen, K. M. (Eds). Aquatic Biodiversity in Latin America (ABLA). Vol 4. Pp: 148-153. Sofia-Moscú: Pensoft Publishers.

Borkent, A. (2009). Dixidae (Meniscus Midges). En Brown, B. V., Borkent, A., Cumming, J. M., Wood, D. M., Woodley, N. E. \& Zumbado, M. A. (Eds.). Manual of Central American Diptera. Vol. 1. Pp: 359-360. Ottawa, Canada: NRC Research Press.

Borkent, A., Spinelli, G. R. \& Grogan, W. L. (2009). Ceratopogonidae (biting midges, purrujas). En Brown, B.V., Borkent, A., Cumming, J. M., Wood, D. M., Woodley, N. E. \& Zumbado, M. A. (Eds). Manual of Central America Diptera. Vol. 1. Ottawa, Canada: NRC Research Press. $714 \mathrm{pp}$.

Chaverri, L.G. (2009). Culicidae (mosquitos, zancudos). En Brown, B.V., Borkent, A., Cumming, J. M., Wood, D. M., Woodley, N. E. \& Zumbado, M. A. (Eds). Manual of Central America Diptera. Vol. 1. Pp: 369-388. Ottawa, Canada: NRC Research Press.
Courtney, G.W. \& Merritt, R. W. (2008). Aquatic Diptera part one: Larvae of aquatic Diptera. En Merritt, R. W., Cummins, K. W. \& Berg, M. B. (Eds.). An In troduction to the Aquatic Insects of North America 4 ed. Pp: 687-722. Dubuque, Iowa, USA: Kendall/Hunt Publishing Company.

Courtney, G., Pape, T., Skevington, J. \& Sinclair, B. (2009). Biodiversity of Diptera. In Foottit, R. \& Adler, P. (Eds.). Insect Biodiversity Science and Society. Pp: 185222. West Sussex, Reino Unido: Blackwell Publishing. https://doi.org/10.1002/9781444308211.ch9

Cranston, P. S. (1995). Introduction. En: Armitage, P. D., Cranston, P. S., Pinder, L. C. V. (Eds.). The Chironomidae. The biology and ecology of non-biting midges. Pp. 297320. Londres, Reino Unido: Chapman \& Hall.

DeWalt, R. E., Resh, V. H. \& Hilsenhoff, W. L. (2010). Diversity and classification of insects and Collembola. En Thorp, J. H. y Covich, A. P. (Eds.). Ecology and classification of North American freshwater invertebrates. Tercera edición. Pp. 587- 657. San Diego, Estados Unidos: Academic Press. https:// doi.org/10.1016/B978-0-12-374855-3.00016-9

Domínguez, E. \& Fernández, H. (2009). Macroinvertebrados bentónicos sudamericanos. Sistemática y Biología. Tucumán, Argentina: Fundación Miguel Lillo. 654 pp.

Ferrington, L. C. (2008). Global diversity of non-biting midges (Chironomidae-Insecta-Diptera) in freshwater. Freshwater Animal Diversity Assessment, 595, 447-455. https://doi.org/10.1007/978-1-4020-8259-7_45

González, C. R., Reyes, C., Jercic, M. I., Rada, V., Saldarriaga, M., Pavletic, C. \& Parra, A. (2016). Manual de culícidos (Diptera: Culicidae) de la zona norte y centro de Chile, incluyendo Isla de Pascua. Segunda Edición. Santiago, Chile: Instituto de salud pública; Ministerio de salud. $95 \mathrm{pp}$.

Grillet, M. E., Villamizar, N. I. Cortez, J., Frontado, H. L., Escalona, M., Vivas-Martínez, S. \& Basanez, M.G. (2005). Diurnal biting periodicity of parous Simulium (Diptera: Simuliidae) vectors in the onchocerciasis Amazonian focus. Acta tropica, 94(2), 139-158. https://doi.org/10.1016/j.actatropica.2005.02.002

Harbach, R.E. 2007. The Culicidae (Diptera) a review of taxonomy classification. Zootaxa, 1668: 591-638. 
Henriques-Oliveira, A., Nessimian, J. \& Dorvillé, L. (2003). Feeding habits of chironomid larvae (Insecta: Diptera) from a stream in the floresta da Tijuca, Río de janeiro, Brazil. Brazilian Journal of Biology, 63(2), 269-281. https://doi.org/10.1590/S1519-69842003000200012

Kazanci, N. \& Ertunç, Ö. (2010). Use of Simuliidae (Insecta: Diptera) species as indicators of aquatic habitat quality of Yeşilırmak River Basin (Turkey). Review of Hydrobiology, 3(1), 27-36.

Lizarralde, M. (2009). Diptera: Generalidades. En: Domínguez, E. \& Fernández, H. R. (Eds.). Macro invertebrados Bentónicos Sudamericanos: Sistemática y Biología. 1 ed. Pp: 341-364. Tucumán, Argentina: Fundación Miguel Lillo.

Marchant, R., Barmuta, L. A. \& Chessman, B. C. (1995). Preliminary study of the ordination and classification of macroinvertebrate communities from running waters in Victoria, Australia. Australian Journal of Marine and Freshwater Research, 45, 945-962. https://doi.org/10.1071/MF9940945

McCafferty, W. P. (1983). Midges, Mosquitoes, Aquatic Gnats and Flies (Order Diptera). In: Aquatic Entomology: The Fishermen's and Ecologists' Illustrated Guide to Insects and Their Relatives. Pp: 283-333. Boston: Jones and Bartlett Publishers.

McCafferty, W. P. (1998). Aquatic Entomology: the fishermen's and ecologist's illustrated guide to insects and their relatives. 1a. ed. Boston: Jones and Barlett Publishers. 448 pp.

Merritt, R.W., Courtney, G. W. \& Keiper, J. B. (2009). Diptera: (Flies, Mosquitoes, Midges, Gnats). En: Vincent, H. R. \& Ring, T. C. (Eds.). Encyclopedia of Insects (Second Edition) (Pp. 284-297). San Diego: Academic Press. https://doi.org/10.1016/B978-0-12-374144-8.00085-0

Merritt, R. W., Cummins, K. W. \& Berg, M. B. (2008). An introduction to the aquatic insects of North America. Dubuque, Kendall/Hunt Publishing Company. $1214 \mathrm{pp}$.

Miranda-Esquivel, D. R. \& Coscarón, S. (2001). Cladistic analysis of Simulium (Trichodagmia) and Simulium (Thyrsopelma) (Diptera: Simuliidae). Zoological Journal of the Linnean Society, 132(4), 429-439. https://doi.org/10.1111/j.1096-3642.2001.tb02469.x
Mosquera-Murillo, Z. \& Córdoba-Aragón, K. E. (2015a). Caracterización de la entomofauna acuática en cuatro quebradas de la cuenca del río San Juan, Chocó, Colombia. Revista de la academia colombiana de ciencias exactas físicas y naturales, 39(150), 67-76. https://doi.org/10.18257/raccefyn.148

Mosquera-Murillo, Z. \& Córdoba-Aragón, K. E. (2015b). Caracterización de la comunidad de macroinvertebrados acuáticos en tres ciénagas de la cuenca media del río Atrato, Chocó, Colombia. Revista Institucional Universidad Tecnológica del Chocó Investigación, Biodiversidad y Desarrollo, 34(1), 22-35.

Mosquera-Murillo, Z. \& Mosquera-Mosquera, M. M. (2017). Diversidad de la entomofauna acuática y calidad deagua en quebradas del río San Juan, Chocó-Colombia. Revista UDCA Actualidad y divulgación Científica, 20(1), 149-161. https://doi.org/10.31910/rudca.v20.n1.2017.72

Mosquera-Murillo, Z. (2017). Insectos acuáticos asociados a raíces de Eichhornia crassipes (MART) Solms en ciénagas del río Atrato, Chocó-Colombia. Revista de Ciencias Univalle, 21(2), 29-44. https://doi.org/10.25100/rc.v21i2.6697

Mosquera-Murillo, Z. (2018). Insectos acuáticos asociados a Eichhornia azurea (Schwartz) Kunth en ciénagas del río Atrato, Chocó-Colombia. Revista Colombiana de Ciencias Animales, 10(1), 15-24.

https://doi.org/10.24188/recia.v10.n1.2018.533

Ocon, C. S. \& Rodrigues, A. (2004). Presence and abundance of Ephemeroptera and other sensitive macroinvertebrates in relation with habitat conditions in Pampean streams (Buenos Aires, Argentina). Archiv für Hydrobiologie, 159, 473-487. https://doi.org/10.1127/0003-9136/2004/0159-0473

Oviedo-Machado, N. \& Reinoso-Flórez, G. (2018). Aspectos ecológicos de larvas de Chironomidae (Diptera) del río Opia (Tolima, Colombia). Revista Colombiana de Entomología, 44(1), 101-109. https://doi.org/10.25100/socolen.v44i1.6546

Paggi, A. C. (2009). Diptera Chironomidae. En: Domínguez, E. \& Fernández, H. R. (Eds.). Macroinvertebrados Bentónicos Sudamericanos: Sistemática y Biología. Pp:383410. Tucumán - Argentina: Fundación Miguel Lillo. https:/ / doi.org/10.5324/cjcr.v0i22.603 
Pérez, S. P. (2007). Muscidae (Diptera) de importancia forense en Colombia: importancia y distribución. En Memorias de Socolen XXXIV Congreso Sociedad Colombiana de Entomología. 250 pp.

Pinder, L. (1986). Biology of freshwater Chironomidae. Annual Review of Entomology, 31(1), 1-23. https://doi.org/10.1146/annurev.en.31.010186.000245

Pinilla, A. (2000). Indicadores biológicos en ecosistemas acuáticos continentales de Colombia: compilación bibliográfica. Bogotá: Fundación Universidad de Bogotá Jorge Tadeo Lozano, Centro de Investigaciones Científicas. $67 \mathrm{pp}$.

Rodríguez, C. V., Quiroz, M. H., Badii, Z. M. \& Solís, R. C. (2004). Riesgos entomológicos asociados a aguas contaminadas. Revista de Salud Pública y Nutrición, 5, 1-8.

Rojas-Cespedes, M. G., Forero-Cespedes, A. M. \& Rei noso-Flores, G. (2018). Dípteros acuáticos (insecta) y su relación con las variables físicas y químicas en un río con bosque seco tropical, Natagaima, Tolima-Colombia. Revista de la Asociación Colombiana de Ciencias Biológicas, 30, 24-37.

Rojas-Sandino, L. D., Reinoso-Flórez, G. \& Vásquez-Ramos, J. M. (2018). Distribución espacial y temporal de dípteros acuáticos (Insecta: Diptera) en la cuenca del río Alvarado, Tolima, Colombia. Biota Colombiana, 19(1), 7091. https://doi.org/10.21068/c2018.v19n01a05

Roldán, G. \& Ramírez, J. J. (2008). Fundamentos de Limnología Neotropical. $2^{\text {a }}$ ed. Medellín: Universidad de Antioquia, ACCEFYN, Universidad Católica de Oriente. 442 pp.

Rosa, B. F. J. V., Dias-Silva, M. V. D. \& Alves, R. G. (2013a). Composition and structure of the Chironomidae (Insecta: Diptera) community associated with Bryophytes in a first-order stream in the Atlantic Forest, Brazil. Neotropical Entomology, 42(1), 15-21. https://doi.org/10.1007/s13744-012-0086-0
Rosa, B. F. J. V., Vasques, M. \& Alves, R. G. (2013b). Chironomidae (Insecta, Diptera) associated with stones in a first-order Atlantic Forest stream. Revista Chilena de História Natural, 86, 291-300. https://doi.org/10.4067/S0716-078X2013000300006

Ruiz-Moreno, J., Ospina-Torres, R. \& Riss, W. (2000). Guía para la identificación genérica de larvas de quironómidos (Diptera: Chironomidae) de la Sabana de Bogotá. II Subfamilia. Caldasia, 22(1), 15-33.

Sandoval, J. C. \& Molina-Astudillo, I. F. (2000). Insectos. En: de la Lanza-Espino, G., Hernández-Pulido, S. \& Carbajal-Pérez, J. L. (Eds.). Organismos indicadores de la calidad de agua y de la contaminación (Bioindicadores). Pp: 412-416. México, D.F. Semarnap, Comisión nacional del agua, PYV, UNAM.

Thorp, J. H. \& Rogers, D. S. (2011). Midges, mosquitoes, blackflies, and other true flies: Insect Order Diptera. En: James, H. T. \& Rogers, D. C. (Eds.). Field Guide to Freshwater Invertebrates of North America. Pp: 247-260. Boston: Academic Press. https:/ / doi.org/10.1016/B978-0-12-381426-5.00027-2

Trivinho, S. (2011). Larvas de Chironomidae: Guía de Identificación (Vol. 2). Sao Carlos. 371p.

Wagner, R., Barták, M., Borkent, A., Courtney, G., Goddeeris, B., Haenni, J. P., Knutson, L., Pont, A., Rotheray, G. E., Rozkosný, R., Sinclair, B., Woodley, N., Zatwarnicki, T. \& Zwick, P. (2008). Global diversity of dipteran families (Insecta Diptera) in freshwater (excluding Simulidae, Culicidae, Chironomidae, Tipulidae and Tabanidae). Hydrobiologia, 595, 489-519. https://doi.org/10.1007/s10750-007-9127-9

Wolff, M. I., Osorio, L. \& Álvarez, G. (1991). Distribución espacial y hábitats larvarios de Aedes aegypti. Boletín Epidemiológico de Antioquia, 15(3), 127-135.

Woodley, N. E. (2009). Athericidae (Athericid flies). En: Brown, B.V., Borkent, A., Cumming, J. M., Wood, D. M., Woodley, N. G. \& Zumbado, M. A. (Eds). Manual of Central America Diptera. Vol. 1. Pp: 491-493. Ottawa, Canadá: NRC Research Press. 


\section{Zuleyma Mosquera Murillo}

Universidad Tecnológica del Chocó

Quibdó, Colombia

zuleymamosquera@gmail.com

https://orcid.org/0000-0001-9029-1013

\section{Sandra Patricia Sánchez Vázquez}

Universidad Tecnológica del Chocó

Quibdó, Colombia

sandrapatricia0220@gmail.com

https://orcid.org/0000-0001-7201-6392

\section{Dípteros acuáticos del departamento del Chocó, Colombia}

Citación del artículo: Mosquera, Z. \& Sánchez, S.P. (2019). Dípteros acuáticos del departamento del Chocó, Colombia. Biota Colombiana, 20(2), 46-58. DOI: 10.21068/ c2019.v20n02a01.

Recibido: 16 de marzo de 2019

Aceptado: 8 de agosto de 2019 